\title{
A STRONG LIMIT THEOREM FOR GAUSSIAN PROCESSES
}

\section{GLEN BAXTER}

1. Introduction and main result. Let $\{W(t), 0 \leqq t \leqq 1\}$ be the Wiener process, that is, a Gaussian stochastic process of real-valued random variables with $E\{W(t)\}=0$ and $E\{W(s) W(t)\}=\min \{s, t\}$. It was discovered independently by Levy [4] and by Cameron and Martin [1] that with probability one

$$
\lim _{n \rightarrow \infty} \sum_{k=1}^{2^{n}}\left[W\left(\frac{k}{2^{n}}\right)-W\left(\frac{k-1}{2^{n}}\right)\right]^{2}=1 .
$$

We demonstrate here a similar result for a large class of Gaussian processes including the Wiener process as a particular case. Notation: in the following $\{X(t), 0 \leqq t \leqq 1\}$ will denote a Gaussian stochastic process of real-valued random variables with mean function $E\{X(t)\}$ $=m(t)$ and covariance function $E\{X(s) X(t)\}-m(s) m(t)=r(s, t)$.

Now assume that $m(t)$ has a bounded first derivative for $0 \leqq t \leqq 1$. Furthermore, assume that $r(s, t)$ is continuous in $0 \leqq s, t \leqq 1$ and has uniformly bounded second derivatives for $s \neq t$. Let

$$
\begin{aligned}
D^{+}(t) & =\lim _{s \rightarrow t^{+}} \frac{r(t, t)-r(s, t)}{t-s}, \\
D^{-}(t) & =\lim _{s \rightarrow t-} \frac{r(t, t)-r(s, t)}{t-s}, \\
f(t) & =D^{-}(t)-D^{+}(t) .
\end{aligned}
$$

The uniform boundedness of the second order derivatives of $r(s, t)$ implies the existence, boundedness, and continuity of the functions $D^{+}(t), D^{-}(t)$, and $f(t)$ over $0<t<1$. In particular $f(t)$ is Riemann integrable over the interval $0<t<1$. The main result can now be stated.

Theorem 1. If $\{X(t), 0 \leqq t \leqq 1\}$ is a Gaussian process satisfying the assumptions of the preceding paragraph, then with probability one

$$
\lim _{n \rightarrow \infty} \sum_{k=1}^{2^{n}}\left[X\left(\frac{k}{2^{n}}\right)-X\left(\frac{k-1}{2^{n}}\right)\right]^{2}=\int_{0}^{1} f(t) d t .
$$

Note that the existence of the first derivative of $r(s, t)$ at $s=t$ is not

Received by the editors May 27, 1955. 
assumed, in fact, it is not wanted. The existence of this derivative would make $f(t) \equiv 0$ and the right-hand side of (2) would reduce to zero, giving an uninteresting result. An important corollary to Theorem 1 holds in case $r(s, t)$ factors into the product of a function of $s$ and a function of $t$. As we shall see later there are many examples of Gaussian processes for which the covariance function has this property.

CoROLlaRy. If $\{X(t), 0 \leqq t \leqq 1\}$ satisfies the assumptions of Theorem 1 , and if

$$
r(s, t)= \begin{cases}u(s) v(t), & s \leqq t, \\ u(t) v(s), & s \geqq t,\end{cases}
$$

then with probability one

(4) $\lim _{n \rightarrow \infty} \sum_{k=1}^{2^{n}}\left[X\left(\frac{k}{2^{n}}\right)-X\left(\frac{k-1}{2^{n}}\right)\right]^{2}=\int_{0}^{1}\left\{u^{\prime}(t) v(t)-u(t) v^{\prime}(t)\right\} d t$.

The proof of Theorem 1 is contained in the next section. In $\$ 3$ we give some examples of the theorem and show that (1) is implied by (2) (and by (4)).

2. Proof of Theorem 1. Let us first assume that $m(t) \equiv 0$. For any positive integer $n$ and integers $j, k$ with $1 \leqq j, k \leqq 2^{n}$ set

Observe that

$$
\begin{aligned}
\Delta X_{k} & =X\left(\frac{k}{2^{n}}\right)-X\left(\frac{k-1}{2^{n}}\right), \\
a_{j k} & =E\left\{\Delta X_{j} \Delta X_{k}\right\}, \\
B_{n} & =\sum_{k=1}^{2^{n}} \Delta X_{k}^{2}, \\
b_{n} & =2 \sum_{j, k=1}^{2^{n}} a_{j k}^{2} .
\end{aligned}
$$

$$
E\left\{B_{n}\right\}=\sum_{k=1}^{2^{n}} a_{k k}
$$

while

$$
E\left\{B_{n}^{2}\right\}=\sum_{k=1}^{2^{n}} 3 a_{k k}^{2}+2 \sum_{j>k=1}^{2^{n}}\left(a_{k k} a_{j j}+2 a_{j k}^{2}\right)
$$

Thus the variance of $B_{n}$ is exactly $b_{n}$. Applying Tchebychev's inequality 


$$
P\left\{\left|B_{n}-E\left\{B_{n}\right\}\right|>n /\left(2^{n}\right)^{1 / 2}\right\}<2^{n} b_{n} / n^{2} .
$$

If we can show that $2^{n} b_{n}$ remains bounded as $n$ becomes infinite, an application of the Borel-Cantelli lemma will show that with probability one $B_{n}-E\left\{B_{n}\right\}$ approaches zero as $n$ becomes infinite.

To estimate $2^{n} b_{n}$, let $M$ be a bound for the three quantities $\left|\partial^{2} r(s, t) / \partial s^{2}\right|,\left|\partial^{2} r(s, t) / \partial s \partial t\right|$, and $\left|\partial^{2} r(s, t) / \partial t^{2}\right|$ in the range $0 \leqq s \neq t \leqq 1$. Using for $r(s, t)$ a Taylor series expansion with remainder it can easily be shown that $j \neq k$ implies

$$
\begin{aligned}
\left|a_{j k}\right| & =\mid r\left(k / 2^{n}, j / 2^{n}\right)+r\left((k-1) / 2^{n},(j-1) / 2^{n}\right) \\
& -r\left(k / 2^{n},(j-1) / 2^{n}\right)-r\left((k-1) / 2^{n}, j / 2^{n}\right) \mid \leqq 3 M(1 / 2)^{2 n} .
\end{aligned}
$$

Also for $k=1,2, \cdots, 2^{n}-1$

$$
\begin{aligned}
a_{k k}= & r\left(k / 2^{n}, k / 2^{n}\right)-2 r\left((k-1) / 2^{n}, k / 2^{n}\right) \\
& +r\left(\left(k-1 / 2^{n}\right),(k-1) / 2^{n}\right) \\
= & (1 / 2)^{n}\left[D^{-}\left(k / 2^{n}\right)-D^{+}\left(k / 2^{n}\right)\right]+O\left((1 / 2)^{2 n}\right)
\end{aligned}
$$

where $2^{2 n} O\left((1 / 2)^{2 n}\right)$ remains bounded independent of $k$ as $n$ becomes infinite. The estimates in (6) and (7) give the boundedness of $2^{n} b_{n}$. In conjunction with (5), the estimate in (7) also implies that

$$
E\left\{B_{n}\right\}=\sum_{k=1}^{2^{n}-1}\left(\frac{1}{2}\right)^{n} f\left(k / 2^{n}\right)+O\left(\left(\frac{1}{2}\right)^{n}\right)
$$

so that $E\left\{B_{n}\right\}$ approaches a limit as $n$ becomes infinite. This limit is exactly the Riemann integral of $f(t)$ from 0 to 1 . Writing

$$
B_{n}=E\left\{B_{n}\right\}+\left[B_{n}-E\left\{B_{n}\right\}\right]
$$

we see that with probability one $B_{n}$ itself converges to the integral of $f(t)$ over $(0,1)$. This finishes the case for which $m(t) \equiv 0$.

If $m(t) \neq 0$, form a new Gaussian process $\{\bar{X}(t), 0 \leqq t \leqq 1\}$ by taking $\bar{X}(t)=X(t)-m(t)$. From the previous arguments we know that with probability one

$$
\lim _{n \rightarrow \infty} \sum_{k=1}^{2^{n}} \Delta \bar{X}_{k}^{2}=\int_{0}^{1} f(t) d t .
$$

Now by Schwarz's inequality with $\Delta m_{k}=m\left(k / 2^{n}\right)-m\left((k-1) / 2^{n}\right)$

$$
\left[\sum_{k=1}^{2^{n}} \Delta m_{k} \Delta \bar{X}_{k}\right]^{2} \leqq \sum_{k=1}^{2^{n}} \Delta m_{k}^{2} \cdot \sum_{k=1}^{2^{n}} \Delta \bar{X}_{k}^{2} .
$$

The second term on the right-hand side of (8) remains bounded with 
probability one. Since $m^{\prime}(t)$ exists and is uniformly bounded over $(0,1)$, the sum of the $\Delta m_{k}^{2}$ goes to zero as $n$ becomes infinite. Thus, with probability one the left-hand side of (8) goes to zero. Finally,

$$
\sum_{k=1}^{2^{n}} \Delta X_{k}^{2}=\sum_{k=1}^{2^{n}} \Delta \bar{X}_{k}^{2}+2 \sum_{k=1}^{2^{n}} \Delta m_{k} \Delta \bar{X}_{k}+\sum_{k=1}^{2^{n}} \Delta m_{k}^{2}
$$

so that

$$
\lim _{n \rightarrow \infty} \sum_{k=1}^{2^{n}} \Delta X_{k}^{2}=\lim _{n \rightarrow \infty} \sum_{k=1}^{2^{n}} \Delta \bar{X}_{k}^{2}=\int_{0}^{1} f(t) d t .
$$

This ends the proof of Theorem 1.

3. Examples. (a) Let us first show that (1) is included in (2) and (4). For the Wiener process $r(s, t)$ factors into a product of a function of $s$ and a function of $t$ like (3); in fact,

$$
r(s, t)= \begin{cases}s, & s \leqq t, \\ t, & s \geqq t .\end{cases}
$$

In the notation of the corollary to Theorem $1, u(s)=s$ and $v(t)=1$ so (4) states that with probability one

$$
\lim _{n \rightarrow \infty} \sum_{k=1}^{2^{n}}\left[X\left(\frac{k}{2^{n}}\right)-X\left(\frac{k-1}{2^{n}}\right)\right]^{2}=\int_{0}^{1} 1 d t=1 .
$$

(b) For a second example ${ }^{1}$ we consider a class of Gaussian processes whose covariance functions are actually Green's functions of certain simple boundary value problems.

It is necessary and sufficient in order that $r(s, t)$ defined for $0 \leqq s$, $t \leqq 1$ be the covariance function of a Gaussian process $\{X(t), 0 \leqq t \leqq 1\}$ that

(i) $r(s, t)=r(t, s)$;

(ii) if $t_{1}, t_{2}, \cdots, t_{n} \in[0,1]$, then $\left(r\left(t_{i}, t_{j}\right)\right)$ is a non-negative definite matrix.

In case $r(s, t)$ has the form (3) where both $u(t)$ and $v(t)$ are nonnegative for $0 \leqq t \leqq 1$, these two conditions can be reduced to just

(iii) if $0 \leqq t_{1}<t_{2} \leqq 1$, then $u\left(t_{2}\right) v\left(t_{1}\right)-u\left(t_{1}\right) v\left(t_{2}\right)$ is non-negative.

To verify this last statement we need only mention that if $r(s, t)$ has the form (3), (i) is automatically satisfied while the determinant of $\left(r\left(t_{i}, t_{j}\right)\right)$ can be explicitly evaluated:

${ }^{1}$ Some of the results here are taken from the author's doctoral dissertation which was written under the guidance of Professor Monroe Donsker at the University of Minnesota. 


$$
\left|\left(r\left(t_{i}, t_{j}\right)\right)\right|=u\left(t_{1}\right) v\left(t_{n}\right) \prod_{i=2}^{n}\left\{u\left(t_{i}\right) v\left(t_{i-1}\right)-u\left(t_{i-1}\right) v\left(t_{i}\right)\right\} .
$$

If (iii) holds the determinant of $\left(r\left(t_{i}, t_{j}\right)\right)$ is non-negative for any choice of $t_{1}, \cdots, t_{n} \in[0,1]$. This shows (see $[5$, p. 103]) that $\left(r\left(t_{i}, t_{j}\right)\right)$ is non-negative definite. On the other hand, if (ii) holds for $r(s, t)$ of the form (3), then the implications of (ii) in the case $0 \leqq t_{1}<t_{2} \leqq 1$ give (iii).

We now show how covariance functions which factor like (3) may come from certain differential systems.

TheOREM 2. Let $p(s)>0, q(s) \geqq 0$, and $p^{\prime}(s)$ be continuous functions on $0 \leqq s \leqq 1$, and let $h$ and $H$ be non-negative extended real-valued numbers. If

$$
\left\{\begin{aligned}
\frac{d}{d s}\left\{p(s) \frac{d y}{d s}\right\}-q(s) y & =0 \\
y(0)-h y^{\prime}(0) & =0 \\
y(1)+H y^{\prime}(1) & =0
\end{aligned}\right.
$$

is incompatible, then the Green's function $r(s, t)$ (see $[2$, p. 304]) is a covariance function of a Gaussian process.

Proof. It is shown in the reference above that the Green's function of system (9) has the form (3) where $u(s)$ and $v(s)$ are linearly independent solutions of the differential equation and satisfy, respectively, the boundary conditions at $s=0$ and $s=1$. We must show that $r(s, t)$ is non-negative and that $u(t) v(s)-u(s) v(t) \geqq 0$ for $s \leqq t$.

To show that $r(s, t)$ is non-negative write

$$
p(s) u^{\prime}(s)-p(0) u^{\prime}(0)=\int_{0}^{e} u(x) q(x) d x .
$$

By the non-negativity of $p(s)$ and $q(s)$ we deduce that $u(s)$ cannot change sign in the interval $0 \leqq s \leqq 1$ and is increasing or decreasing according as it is positive or negative. Similarly we deduce that $v(s)$ does not change sign in the interval $0 \leqq s \leqq 1$ and is increasing or decreasing according as it is negative or positive. Suppose for a while that $u(t) v(s)-u(s) v(t)$ is positive whenever $t>s$. If $u(s) v(t)$ is negative, then either $u(s)$ is positive and $v(t)$ is negative or vice-versa. Since either case is handled in the same way we consider only $u(s)$ positive and $v(t)$ negative. In that case $t>s$ implies $u(t) v(t) \leqq u(s) v(t)$ $\langle u(t) v(s)$ which contradicts the fact that $v(t)$ is increasing in $t$. Thus $u(s) v(t)$ is non-negative for $0 \leqq s, t \leqq 1$.

To prove that $u(t) v(s)-u(s) v(t)$ is positive for $t>s$ we write 


$$
\begin{aligned}
p(t)\left\{u^{\prime}(t) v(s)-u(s) v^{\prime}(t)\right\}- & \\
& =\int_{0}^{t} q(x)\{u(x) v(s)-v(x) u(s)\} d x .
\end{aligned}
$$

For $t$ in the neighborhood of $s$ the right-hand side of (10) is very small. Thus the derivative of $u(t) v(s)-u(s) v(t)$ with respect to $t$ (fixed but arbitrary $s$ ) is positive for $t>s$ so that the positivity of $u(t) v(s)-u(s) v(t)(t>s)$ is verified. This completes the proof of the theorem.

Now let $\{X(t), 0 \leqq t \leqq 1\}$ be a Gaussian stochastic process whose covariance function is the Green's function of (9). By the definition of the Green's function $u^{\prime}(t) v(t)-u(t) v^{\prime}(t)=1 / p(t)$ so that the corollary to Theorem 1 states that

$$
\lim _{n \rightarrow \infty} \sum_{k=1}^{2^{n}}\left[X\left(\frac{k}{2^{n}}\right)-X\left(\frac{k-1}{2^{n}}\right)\right]^{2}=\int_{0}^{1} \frac{1}{p(t)} d t
$$

with probability one. For an explicit example take the system (9) where $p(s)=1$, and $q(s)=h=H=0$. The system is incompatible and the Green's function is

$$
r(s, t)= \begin{cases}s(1-t), & s \leqq t, \\ t(1-s), & s \geqq t .\end{cases}
$$

The Gaussian process with this covariance function and mean function zero has been the subject of considerable study by Doob [3] and others. We have immediately for this process

$$
\lim _{n \rightarrow \infty} \sum_{k=1}^{2^{n}}\left[X\left(\frac{k}{2^{n}}\right)-X\left(\frac{k-1}{2^{n}}\right)\right]^{2}=\int_{0}^{1} \frac{1}{1} d t=1 .
$$

It is easy to see that the Wiener process is another explicit example of Theorem 2 and (11).

\section{REFERENCES}

1. R. H. Cameron and W. T. Martin, The behavior of measure and measurability under a change of scale in Wiener space, Bull. Amer. Math. Soc. vol. 53 (1947) pp. 130137.

2. R. Courant and D. Hilbert, Methoden der Mathematischen Physik, vol. 1, Berlin, Springer, 1931.

3. J. L. Doob, Heuristic approach to the Kolmogorov-Smirnov theorems, Ann. Math. Statist. vol. 20 (1949) pp. 393-403.

4. P. Levy, Le mouvement brownien plan, Amer. J. Math. vol.62 (1940) pp. 487-550.

5. S. Perlis, Theory of matrices, Cambridge, Addison-Wesley, 1952.

MassachusetTs Institute of TeChNology 